\title{
VON DEN URAUSTRALIERN
}

\author{
DONALD F. THOMSON
}

Mit Farbbild

Der australische Eingeborene ist der gutmütigste, aber auch verkannteste aller Menschen. Ich möchte seinem Andenken gerecht werden; denn die Lebensweise, die unvorstellbar komplizierte Gesellschaftsordnung, die hohe Ethik und die strengen Sittengesetze in seiner Stammeskultur sind am Verschwinden und werden bald nur noch in Erinnerungen leben.

Man bezeichnete die australischen Ureinwohner als die primitvsten noch lebenden Vertreter der Menschenrassen dieser Erde. Und man liest immer wieder, wenigstens in der frühen Literatur über Australien und seine Eingeborenen, daß diese auf der untersten Stufe der Menschheit stehen oder demoralisiert seien; eine klare Begründung dieser Behauptung wird nicht gegeben.

Soviel auch über die Uraustralier geschrieben wurde, fast nie wurden ihre positiven Eigenschaften erwähnt: die tiefe Religiosität, die Betonung des Geistigen gegenüber dem Materiellen, die Geringschätzung von Reichtum und Besitz und das mystische Weltbild, das in ihrem Verhältnis zur Natur, besonders zu den Vögeln und den Tieren, so gut zum Ausdruck kommt und in dem ihre Sagen und ihre totemistischen Glaubensvorstellungen verankert sind.

Entgegen der allgemeinen Überzeugung ist der australische Eingeborene nicht bequem oder faul, solange er sein durchorganisiertes Stammesleben in seinem Klan lebt: ein Zustand, der heute in Australien leider fast ganz verschwunden ist. Abgesehen von der Freundlichkeit der Uraustralier allen gegenüber, die ihr Verhalten zu verstehen suchen und selbst befolgen, fällt dem Besucher eines Eingeborenenlagers zuerst die herrschende Ordnung und die harte Arbeit auf. Jedes Glied der Gruppe, Männer und Frauen, nimmt teil an der Jagd und der Sammeltätigkeit. Wenn die Schatten in der späten Nachmittagssonne länger werden, kehren sie von der systematischen Nahrungssuche in Frauen- und Männergruppen oder familienweise ins Lager zurück; die Männer von der Känguruhjagd oder vom Fischen, die Frauen vom Sammeln der Früchte, Knollen und anderer Pflanzenteile und von der Kleintierjagd. Wenn sie sich mit ihrer Tagesernte dem Lager nähern, lesen die Frauen große Bündel Holz zusammen und tragen diese auf den Schultern oder balancieren sie auf einem aus Rinden oder aus Pandanusblättern sauber verfertigten runden Polster auf dem Kopf. Bald herrscht im Lager eine Atmosphäre der Geschäftigkeit und Zusammenarbeit: nicht nur innerhalb der Familien am eigenen Feuer, sondern auch unter Einzelnen in größeren Gruppen.

Die Verteilung der Arbeit unter die Geschlechter und die bereitwillige, gute $\mathrm{Zu}$ sammenarbeit bei allen gemeinsamen Unternehmungen überraschen jeden Weißen, der diese Menschen nur vom Hörensagen oder aus Büchern kennt. Eine geplante Treibjagd, bei der das Spinifexgras angezündet wird und das Feuer das Wild den in offener Landschaft wartenden Jägern zutreibt, oder ein groß aufgezogener Fischfang, an dem oft auch Frauen teilnehmen, wird ihm zu einem unvergeßlichen Erlebnis.

Der reibungslose Ablauf gemeinschaftlicher Unternehmungen wird erst richtig verständlich, wenn man diese Eingeborenen besser kennenlernt. Diese Zusammenarbeit steht und fällt mit einer Gesellschaftsordnung und Verwandtschaftsgliederung, die das korrekte Verhalten genau festlegen. Das Verwandtschaftssystem, das von den australischen Ureinwohnern stark entwickelt wurde, ist eigentlich eine Ausdehnung der Verwandtschaftsgrade innerhalb einer Familie mit den entsprechenden traditionellen und bindenden Verhaltensregeln auf Menschen, die wir nicht zu unseren Verwandten zählen würden. Dieses System ist für den australischen Eingeborenen eine Selbstverständlichkeit und in seinen Glaubensvorstellungen verankert, nach denen alle Mitglieder des 


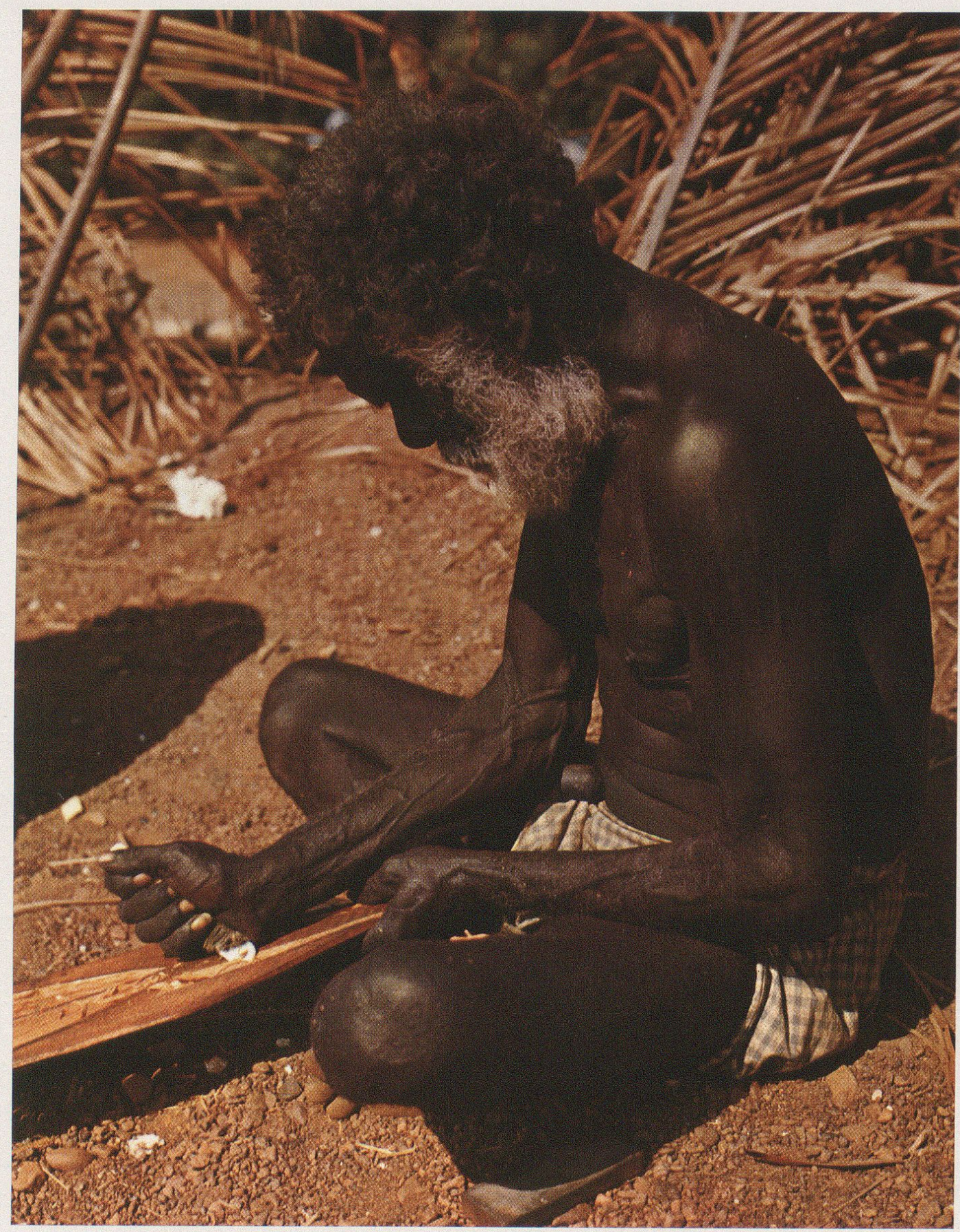

Eingeborener der Bathurst-Insel schneidet sich mit einem selbstgemachten Schnitzmesser einen Zeremonienspeer. 


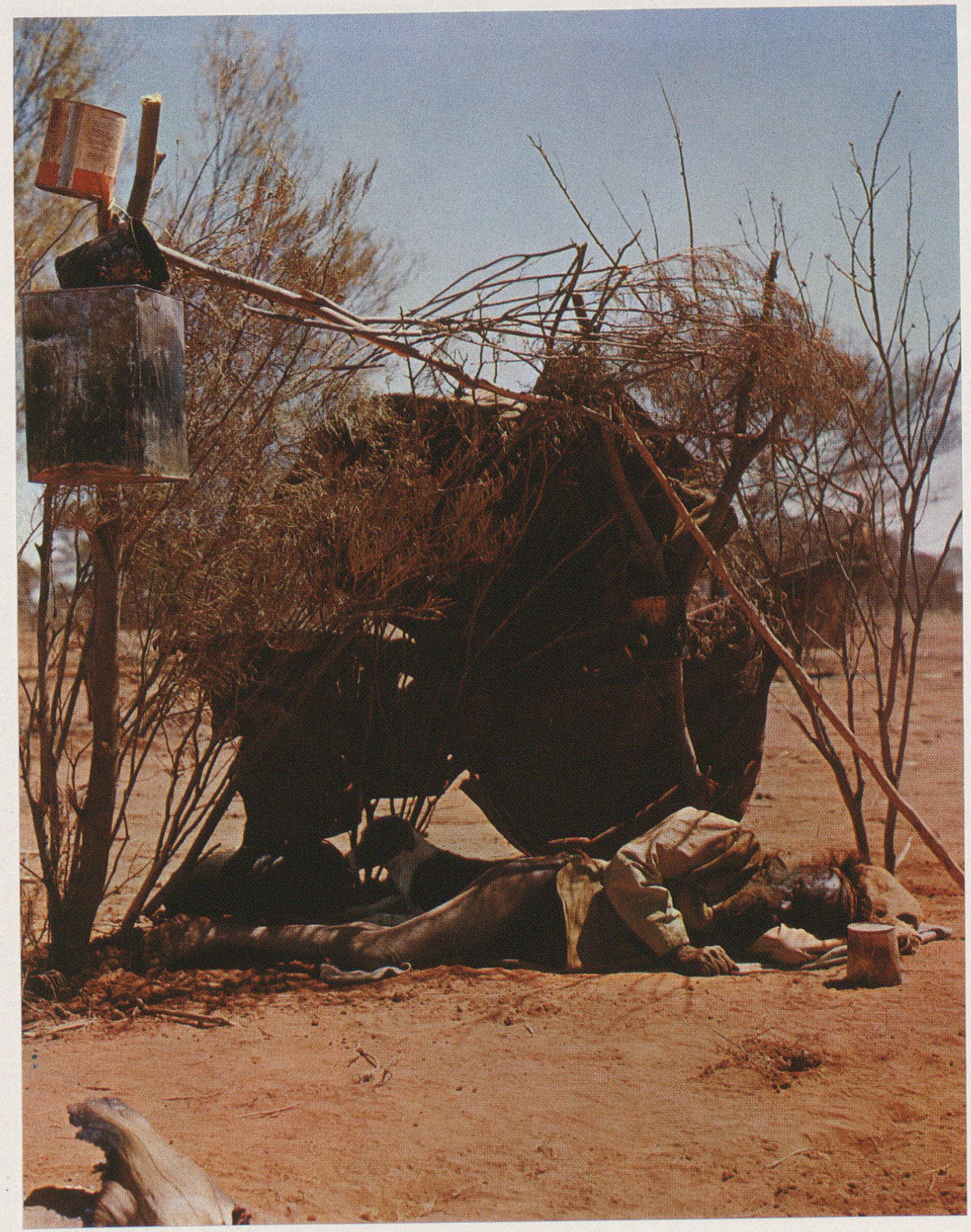

Haus eines älteren Australiers. Die älteren Ureinwohner weigern sich, in den von der Regierung erstellten Hütten zu leben. Sie ziehen die primitiven Unterkünfte aus Zweigen, Säcken und Wellblech vor. 
Klans, in den er geboren wurde, durch einen gemeinsamen totemistischen Vorfahren miteinander verwandt sind. In einem Verwandtschaftssystem, bei dem die Verwandtschaftsbeziehungen der eigenen Familie unbeschränkt auf alle anderen Mitmenschen erweitert werden, wird ein Mann die Bezeichnung «Vater» für jeden anwenden, den sein Vater «Bruder» nennt, und er wird allen «Schwestern» (wirklichen und klassifikatorischen) seiner Mutter auch «Mutter» sagen. Alle Söhne und Töchter seiner «Väter» und «Mütter» sind also im erweiterten Sinne seine «Brüder» und «Schwestern».

Korrektes Benehmen gegenüber jedem Mitglied der Horde, in der sie leben, wird den Kindern schon in den ersten Jahren beigebracht. Sie müssen ebenfalls lernen, dieses Benehmen auf alle Menschen, die ihnen begegnen, anzuwenden. Auch auf Befolgung des korrekten Verhaltens und der Etikette beim Besuch eines fremden Lagers oder bei wichtigen zeremoniellen Anlässen wird viel Wert gelegt. Die aufmerksame Wahrung dieser Formen ist sehr wichtig, wenn man die Eingeborenen als Freunde gewinnen will. Die vielgeschmähten Uraustralier legen weit mehr Wert auf das, was wir «gute Kinderstube»nennen, als manche Europäer.

Als die ersten Weißen nach Australien kamen, fanden sie den Kontinent schon bevölkert von einer dunkelhäutigen Rasse, deren körperliche Beschaffenheit und Lebensweise außerordentlich primitiv waren. Diese Menschen waren nackt. Nur in wenigen Gebieten, wie den kalten Regenwäldern im Süden des heutigen Staates Victoria, verfertigten sich die nun ausgestorbenen Eingeborenen Decken aus Fellen der Känguruhs und anderer Tiere. Sie bauten keine Häuser, besaßen keine Gärten und außer dem halbwilden Dingo, einem gelbbraunen, wolfähnlichen Tier mit aufstehenden Ohren und buschigem Schwanz, keine Haustiere. Von Landwirtschaft und Viehzucht wußten sie nichts und hatten daher keine zuverlässige Nahrungsquelle. Als Wildbeuter waren sie gezwungen, ihr Gebiet zu durchwandern. Ein seßhaftes «Dorfleben» kam für sie nicht in Frage. Der Umstand, daß sie keinen Landbau betrieben und keine Tiere züchteten, war oft Anlaß abfälliger Berichte über die Uraustralier. Man darf aber nicht vergessen, daß auch die Weißen mit ihrem wissenschaftlichen Können und ihren Erfahrungen bis heute kein einziges einheimisches Tier fanden, das sich zur Züchtung eignen würde, und auch keine einzige zum Anbau geeignete Pflanze, außer dem Queensland-Nußbaum (Macadamia), dessen Nüsse die Eingeborenen schon lange kannten.

Als die ersten Siedler eintrafen, war den Eingeborenen das Eisen noch nicht bekannt. Noch immer bedienten sie sich der Waffen und Geräte aus Stein, Holz oder Knochen, manche roh verfertige und grob, andere von reinstem handwerklichem Können zeugend. Die Uraustralier stellten ihre Steingeräte in der Abschlagtechnik her, wie sie aus dem Altpaläolithikum in Europa und anderen Teilen der Welt bekannt sind; aber gleichzeitig arbeiteten sie auch mit Schleifen und Polieren, der Technik des Neolithikums, hauptsächlich bei der Herstellung ihrer scharfkantigen Äxte, jener klobigen, rauhen Hack- und Schabgeräte, wie sie der erste Weiße zu sehen bekam.

Trotz ihrer primitven äußerlichen Erscheinung und Lebensweise waren die Uraustralier meistens freundlich und dem Fremden gut gesinnt.

In meinem ganzen Studium, in all den Textbüchern und Monographien war nichts, das mich auf die erste Begegnung mit diesen Eingeborenen vorbereitet hätte. Wie so manche andere Anthropologen, stellte ich mir die Uraustralier stets als nomadische Jäger vor, die in den dürren, unwirtlichen Gegenden im Inneren des Kontinentes einen harten Kampf ums Dasein führten. So war ich denn erstaunt, mich mitten in einer Schar von Fischern zu finden, die Kanus herstellen, sich hauptsächlich vom Ertrag des Meeres ernährten, wo sie mit Harpunen, mit mehrspitzigen Speeren und mit Netzen fischten und mit Pflanzengiften die Fische im untiefen Wasser der Lagunen und in den stilleren Gewässern der Flüsse betäubten. Diese Menschen wagten große Fahrten in die Gewässer der Princess Charlotte Bai auf der Suche nach verschiedenartigen Schildkröten und nach der Seekuh (Dugong), einem warmblütigen Säugetier, nah verwandt mit 
den Walen. Auf dem Bugrand ihres Kanus stehend, harpunierten sie ihre Beute. Die Fisch-, Schildkröten- und Seekuhnahrung ergänzten sie mit den Eiern von Schildkröten und Vögeln, welche sie auf den vor der Küste liegenden Riffen und Inseln sammelten. Aber auch alle möglichen Pflanzen und Pflanzenteile gehörten zur Mahlzeit. Bei stürmischem Wetter suchten die Frauen in der nahen, geschützten Flußmündung nach Schaltieren. Sie sammelten Pflanzen, zum Beispiel Jamwurzeln, die im feuchten Dschungel in der Nähe der Küste wild gediehen, und auch manche Früchte und die Eier von Buschhühnern und Busch-Tale-Galahühnern (Großfußhühner oder Wallnister), je nach der Jahreszeit.

All dies erkannte ich nicht sogleich. Es war ein Teil der Erfahrungen, die ich unter diesen Eingeborenen sammelte, als ich nach und nach ihre Freundschaft gewann und in ihre Lager aufgenommen wurde und mich unter ihnen niederlie $\beta$, um wie sie zu leben. So begannen die langen Jahre, die ich unter den letzten nomadischen Jägern von Cape York und anderen Gebieten Australiens verbrachte.

\section{SHOWA-SHINZAN \\ Der jüngste Vulkan im südlichen Hokkaido, Japan}

OSKAR BÄR

Die Geburt des Vulkans Paracutin in Mexico fand bei uns große Beachtung. Es erschienen verschiedene Publikationen darüber, und ein ausgezeichneter Schulfilm (SAFU 3o1) entstand.

In unseren Geographenkreisen recht unbemerkt verlief hingegen die nicht minder faszinierende Eruption des Usu-Vulkans auf Hokkaido in den Jahren 1943 bis 1945. Sie führte zur Bildung des Shôwa-Shinzan. Das japanische Wort «Shinzan» bedeutet «neuer Berg» (san = Berg; z. B. Fuji-san). "Shôwa» ist der Name des heutigen Kaiserhauses (1926- -000 ). "Shôwa-Shinzan» besagt folglich: neuer Berg der Shôwa-Aera. Desgleichen heißt ein 1910 entstandener Vulkankegel der gleichen Region Meiji-Shinzan (Meiji-Aera: 1868-1912).

Auf einer zehntägigen Exkursion ins Vulkangebiet Süd-Hokkaidos im Frühjahr 1964 hatte ich Gelegenheit, diese interessanten Erscheinungen zu studieren. Aus den Gesprächen mit Prof. Dr. T. Ishikawa, einem führenden japanischen Vulkanologen, der mich auf der Exkursion begleitete, und mit Hilfe seiner Publikationen habe ich diesen kurzen Bericht zusammengestellt. Herrn Prof. Ishikawa sei an dieser Stelle herzlich gedankt.

Der Vulkan Usu liegt etwa $30 \mathrm{~km}$ NNW der Stadt Muroran in Süd-Hokkaido, zwischen der Funka-Bay (Eruptions-Bucht) und dem Tôya-See (vulkanischer CalderaSee). Er bedeckt eine Fläche von rund $30 \mathrm{~km}^{2}$ (Durchmesser ca. $6 \mathrm{~km}$ ), und sein höchster Punkt, der Usu-Dom, erreicht 725 m über Meer.

Die vulkanische Tätigkeit begann beim Usu als Folge des Caldera-Einbruchs (zusammen mit der Entstehung des Tôya-Nakajima im Zentrum des Caldera-Sees) gegen Ende des Pleistozäns. Sie dauert bis heute an. Bekannte Eruptionen fanden in folgenden Jahren statt: 1663 (Kanbun Aera), 1768 (Meiwa Aera), 1822 (Bunsei Aera), 1853 (Kaei Aera) und 1910 (Meiji-Aera). Der Vulkan baut sich zur Hauptsache aus verschiedenen andesitischen Laven auf. Wichtig sind auch Aschenlagen und mächtige Bimssteinschichten, die oft deutlich erkennbare Plateaus bilden.

Der neuste Ausbruch im Jahre 1943 ereignete sich am Osthang des Hauptvulkans, etwa zwei Kilometer vom Usu-Dom entfernt, wenig westlich des Dorfes Fukaba. Der ganze Ablauf des Geschehens läßt sich in drei Abschnitte einteilen: 